\title{
ACTUALIDAD INVESTIGATIVA Y PERSPECTIVAS DE LA LINGÜÍSTICA COGNITIVA: TIEMPO, ESPACIO Y METÁFORA*
}

\section{Luis Herrera Vázquez **}

\section{Resumen}

El doctor Javier Valenzuela es uno de los investigadores más importantes a nivel mundial en lingüística cognitiva. En esta entrevista profundiza sobre los alcances generales de esta ciencia, repasando la relevancia de la corporeización y la metáfora en la comunicación humana, además las investigaciones pioneras que desarrolla en el laboratorio Red Hen. A futuro, subraya el entrevistado, uno de los grandes desafíos es afinar los métodos de estudio de la metáfora.

Palabras clave: lingüística cognitiva, corporeización, metáfora, comunicación.

\section{NewsinVESTIGATIVE AND PROSPECTS IN COGNITIVE LINGUISTICS: TIME, SPACE AND METAPHOR}

\begin{abstract}
Dr. Javier Valenzuela is one of the most important researchers worldwide in cognitva linguistics. In this interview elaborates on the general scope of this science, reviewing the relevance of the embodiment and metaphor in human communication also develops the pioneering research in the lab Red Hen. In the future, says the respondent, one of the great challenges is to refine the methods of study of metaphor.

Keywords: cognitive linguistics, embodiment, metaphor, communication.
\end{abstract}

Recibido: 25-08-2015

Aceptado: 23-03-2016

* Entrevista que surge de la investigación interna de la carrera de Pedagogía en Lengua Castellana y Comunicación de la Universidad Autónoma de Chile, incorporada dentro del financiamiento Plan de Mejora-Vinculación con el Medio: "Nuevos avances en lingüística cognitiva y neurolingüística" (2014-2015).

** Chileno. Magíster en docencia Universitaria y Diplomado en Lingüística Aplicada. Profesor de Castellano. Académico Universidad Autónoma de Chile, Talca, Chile. luis.herrera@gmail.com 


\section{Presentación}

El Doctor Javier Valenzuela Manzanares ha desarrollado una sobresaliente carrera en los estudios del lenguaje y las ciencias cognitivas desde la Universidad de Murcia y otras instituciones. Participando como autor, articulista o mencionado como investigador, está presente en los libros más importantes de lingüística cognitiva que se han publicado en Hispanoamérica. De hecho, durante el presente año colabora con Iraide Ibarretxe en la publicación del libro Lingüística y cognición: el lenguaje desde la lingüística cognitiva (Editorial Síntesis).

Entre sus líneas de investigación destacan la gramática de construcciones, validaciones psicolingüísticas de la lingüística cognitiva y las metáforas del tiempo y el espacio. Actualmente, también colabora con el Red Hen Lab de la UCLA que desarrolla estudios sobre la comunicación multimodal, junto a destacados investigadores como Mark Turner y Francis Steen.

La entrevista* se ha organizado en cuatro apartados que parten desde la conceptualización general de la lingüística cognitiva y finalizan con las investigaciones y desafíos puntuales del entrevistado. En el primero de ellos, "La lingüística cognitiva como paradigma" el Dr. Valenzuela discute sobre la revolución cognitiva de Chomsky y la consolidación de la lingüística cognitiva como ámbito de investigación relevante. En el segundo, “Objetividad, cultura y corporeización" el entrevistado se adentra en las particularidades de la metáfora y la construcción particular del significado vinculado con aspectos trascendentales como la cultura y la corporeización, importantes en nuestro conocimiento lingüístico. En el tercero, "Últimas investigaciones y laboratorio Red Hen Lab" el Dr. Valenzuela explica los avances más significativos en los estudios de la metáfora y da cuenta de las ventajas claves de lo desarrollado en el Red Hen Lab de la UCLA, con tecnología que permite pesquisar evidencia audiovisual pertinente para el estudio lingüístico. Finalmente, en el cuarto apartado, "Desafíos futuros", se discute sobre los alcances metodológicos para afinar los estudios de metáfora, la necesidad de generar mayor comunicación con otras disciplinas y se presenta la complejidad de identificar la correspondencia entre elementos neuronales específicos y elementos lingüísticos puntuales.

* Realizada durante el primer semestre de 2015. 


\section{La lingüística cognitiva como paradigma}

L. Herrera V.: Considerando a Noam Chomsky como el punto de inflexión hacia el desarrollo de las ciencias cognitivas ¿Realmente se han dimensionado sus aportes al desarrollo de la ciencia, atendiendo a la cantidad de refutaciones posteriores sobre todo en el área de la lingüística?

J. Valenzuela M.: La importancia de Chomsky en el desarrollo de la lingüística y de las ciencias cognitivas no puede ser en ningún momento negada ni minusvalorada. Su aportación fue crucial para el avance de la lingüística y, de hecho, marcó el principio de lo que se conoce como "ciencia cognitiva”. A finales de los años '50 del siglo pasado, Chomsky jugó un papel esencial en la introducción de una manera más moderna de acercarse a las ciencias cognitivas, demostrando con su crítica al acercamiento conductista de Skinner a la lengua que era necesario postular estructuras mentales, abrir la "caja negra" de la mente e ir más allá de la simple alineación de estímulo-respuesta. Hay que reconocer, sin embargo, que una gran parte de sus ideas originales han ido siendo muy cuestionadas, empezando por la misma hipótesis de la gramática universal.

Por ejemplo, un gran número de seguidores suyos abandonaron la visión transformacional de la gramática a partir de los años 70 (cuando se realizaron experimentos que pusieron en duda su utilidad como modelo psicológicamente realista del procesamiento lingüístico humano). En realidad, sus ideas han ido evolucionando y hasta cierto punto, reduciéndose. También creo que hay un cierto "hermetismo" en sus últimas teorías, de manera que no está completamente claro qué es lo que postula la teoría chomskiana (y hay que acudir a "interpretadores" de lo que Chomsky en realidad quiere decir, lo que resulta un modelo extraño para una explicación científica). Personalmente, encuentro muy difíciles de aceptar algunos de sus postulados, como por ejemplo su énfasis en negar la centralidad de la función comunicativa del lenguaje, favoreciendo en vez de ello la función representativa. En mi opinión, intentar explicar la estructura y el funcionamiento del lenguaje sin tener en cuenta su función comunicativa es algo completamente erróneo. La dimensión social no puede en ningún momento abandonarse.

L.H.V: Reconociendo que existen asociaciones de lingüística cognitiva, libros capitales como el suyo con la Dra. Ibarretxe-Antuñano, Cuenca 


\section{y Hilferty o que existen investigadores tan afamados como Fauconnier,} Turner, Langacker, Talmy o Lakoff, todavía existen voces que discuten si la lingüística cognitiva es realmente una teoría unificada, ¿Qué avances han permitido o permitirán que sea considerado un paradigma teórico irrefutable y de manera unánime?

J.V.M.: La solidez de la Lingüística Cognitiva como acercamiento es ya innegable: ha permeado todos los ámbitos y debe ser considerado como un paradigma establecido a todos los niveles. No sólo hay revistas especializadas (Cognitive Linguistics lleva ya un cuarto de siglo publicándose y tiene una posición destacada entre las revistas de lingüística con índice de impacto; la revista Review of Cognitive Linguistics también tiene muy buena posición y calidad), sino que investigaciones que partan o utilicen supuestos de lingüística cognitiva se publican en prácticamente la totalidad de revistas de ciencia cognitiva en general. En este sentido, al confluir la lingüística cognitiva con asuntos centrales de la ciencia cognitiva (como los trabajos de cognición corporeizada o los acercamientos más estadísticos o "basados en el uso" de la gramática de construcciones), realmente podemos ver la influencia de esta corriente en todos sitios. El volumen de información que se publica (en libros, capítulos de libro, artículos, tesis doctorales, de master, etc.) es ingente. El número de asociaciones nacionales y de congresos, tanto internacionales como nacionales, tanto generalistas como de acercamientos específicos de la teoría, es también increíblemente numeroso. En este sentido, no cabe la menor duda de que la lingüística cognitiva es un paradigma completamente establecido, que tiene cosas que ofrecer a todos los ámbitos de los estudios del lenguaje, desde los más descriptivos y gramaticales a los de segundas lenguas, del procesamiento del lenguaje natural (NLP), psicolingüísticos, neurolingüísticos, sociolingüísticos, literarios, culturales, etc.

Otro asunto a discutir es la naturaleza del paradigma. Desde un principio se ha reconocido a la lingüística cognitiva como una "corriente", un "acercamiento" más que una teoría absolutamente concreta. Esto no tiene por qué ser algo negativo. La lingüística cognitiva se puede tomar como una serie de teorías concretas, o bien como un tipo de acercamiento al lenguaje que lo relaciona con otras facultades cognitivas. Indudablemente, al ser un paradigma tan activo y en ebullición, existen multitud de afirmaciones y teorías; algunas de ellas pasarán el test del tiempo y otras tendrán que ser moduladas o incluso abandonadas. En 
general, en la ciencia hay pocas cosas "irrefutables", casi por definición de lo que es ciencia. Y la unanimidad tampoco es una medida muy fiable, porque prácticamente nunca se va a alcanzar (es una cuestión más de grado que absoluta). Pero sí que estoy convencido de que las ideas básicas de la lingüística cognitiva ya han permeado la ciencia cognitiva y están siendo asumidas por un gran número de investigadores de la cognición, muchos de los cuales ni siquiera se dedican a la lingüística. El objetivo de una teoría o un acercamiento, además, no debe ser "tener razón" o ganar adeptos, sino proponer una serie de ideas que sirvan de acicate o de estímulo para hacer avanzar nuestra comprensión de cómo funcionan las cosas. $\mathrm{Y}$ ese es un proceso que claramente no tiene fin. Siempre estaremos intentando alcanzar explicaciones más completas y adecuadas. La lingüística cognitiva ha permitido encauzar muchos esfuerzos en una dirección que se ha probado muy efectiva y que ha logrado un nivel de convergencia entre las distintas ciencias cognitivas que es muy deseable.

\section{Objetividad, cultura y corporeización}

L.H.V: Pasando a otro punto de la lingüística cognitiva, si se evidencia que, por ejemplo, la percepción del tiempo está relacionado con la corporeización subjetiva, así como múltiples otras concepciones de la realidad, ¿La lingüistica cognitiva subraya la imposibilidad de un conocimiento objetivo?

J.V.M.: Todo depende de qué se considere un "conocimiento objetivo". El relativismo conceptual que defiende la lingüística cognitiva está siempre muy sujeto a condiciones. La lingüística cognitiva no aboga por un relativismo extremo como podría encontrarse en el postestructuralismo de Derrida, Lacan o Foucault. Lo que la lingüística cognitiva sí dice es que no existen conceptos "objetivos" en el sentido de "absolutos" o "independientes del conceptualizador". Para empezar, todos nuestros conocimientos, nuestros conceptos, deben ser considerados como "interactivos": surgen de la interacción de nuestras mentes/cuerpos con nuestro ambiente. Un ejemplo muy básico y claro: el concepto de "rojo" no existe de manera "objetiva" e independiente del observador. Este concepto existe para nosotros, como humanos, debido a especificidades de nuestra biología y fisiología: tenemos ciertas células nerviosas en nuestros ojos que han evolucionado para reaccionar frente a la presencia 
de una determinada longitud de onda en la luz; son capaces de detectar en el espectro lumínico este componente y envían activación al cerebro que nos informa de que estamos "viendo rojo". Los colores que percibimos en el mundo son, para empezar, dependientes de esas células nerviosas oculares (los conos y los bastones), que nos proporcionan información útil para nuestra especie. Nosotros no podemos percibir variaciones en longitud de onda de la luz que sean superiores a la del color violeta o inferiores a la del color rojo (de ahí lo de "infrarrojo" o "ultravioleta", que cae fuera del espectro perceptible por nosotros). Otras especies animales perciben otro segmento de radiación lumínica diferente, con lo que para empezar, conceptos cromáticos como el rojo o el verde son "objetivos" en el sentido de "existentes en la naturaleza de manera independiente del observador". Si extraemos esta información del mundo es porque nos ha resultado útil evolutivamente hablando (por ejemplo, podemos distinguimos si una fruta está madura o no según su color).

L.H.V.: ¿Y cuál es el papel de la cultura en consideración a la clásica discusión entre factores externos e innatismo?

J.V.M.: Por supuesto, a todo esto hay que añadir inmediatamente el rol de la cultura, que es completamente básico. Como es bien sabido, las diferentes lenguas y culturas segmentan este espacio cromático que podemos percibir: lenguas como el ruso o el italiano establecen diferencias entre colores como siniy y goluboy (ruso) o blu y azurro (italiano), que para el español no son más que variantes de un sólo color: el azul (oscuro o claro). Es otra manera en la que el concepto de color es relativo: hay relatividad biológica y hay relatividad cultural. La influencia de la cultura en el establecimiento de distinciones culturales está absolutamente demostrada. Como hemos dicho, una idea sería la de "el cerebro propone y la cultura escoge", aunque es posible que esta sea una manera ligeramente simplista de conceptualizar el problema, aunque creo que útil. Vista la cuestión desde este punto de vista, la afirmación de que la lingüística cognitiva "subraya la imposibilidad de un conocimiento objetivo" puede entonces matizarse y ponerse en su contexto adecuado.

L.H.V.: En consecuencia, y siempre desde la lingüística cognitiva, ¿Qué podemos inferir actualmente sobre la antigua y discutida relación lengua-cultura-cerebro?

J.V.M.: Este es un tema muy estudiado por su gran interés. Existe más o menos un consenso entre un gran número de investigadores en que 
existe una gran interrelación entre lengua, cultura y cognición y existe una gran cantidad de estudios que investigan su relación. Si tuviéramos que resumir una de las ideas más populares, podría ser algo así como "el cerebro propone y la lengua/cultura escoge". Por ejemplo, Dolscheid et al. (2013) investigaron las diferentes metáforas espaciales que se utilizan para hablar de la altura tonal, lo agudo o grave que es un sonido. En algunos idiomas como el inglés y el español, la metáfora espacial para describir esto es la verticalidad: un sonido agudo es "alto" y un sonido grave es "bajo". En otros idiomas, esta distinción sonora se establece con la metáfora "grueso-delgado" (o "gordo-fino"): los sonidos graves son gruesos y los sonidos agudos, finos. ¿De dónde sale esta variación entre lenguas? Estos investigadores comprobaron que bebés de 4 meses de edad eran sensibles a ambos tipos de asociación: los bebés miraban más tiempo a una pelota en una pantalla que iba subiendo mientras escuchaban un sonido "ascendente" (que se hacía más agudo), frente a casos en los que la pelota subía mientras el sonido se iba haciendo más grave. Lo mismo encontraron cuando los bebés miraban una línea que se iba haciendo más ancha o más estrecha al tiempo que sonaba un sonido que se hacía más agudo o más grave: en este caso, la asociación preferida es que el sonido se vaya haciendo más grave a medida que la línea se hace más ancha y al revés. La idea que se extrae de estas investigaciones es que estas asociaciones concretas no son entonces debidas a la cultura o la lengua, sino que están proporcionadas manera más o menos innata y universal por nuestros sesgos cognitivos. Luego, la lengua y la cultura "eligen" por así decir, entre las posibles asociaciones y potencian una de ellas, que es la que pasa a formar parte del repertorio lingüístico y finalmente, de la estructura mental del adulto. Es conocida la influencia que un aspecto puramente cultural, como es la dirección de escritura/ lectura, tiene sobre la organización espacial del tiempo en el eje lateral. Los hablantes de lenguas que se escriben de izquierda a derecha (como el español o el inglés) tienden a situar el pasado a la izquierda y el futuro a la derecha, mientras que los pertenecientes a culturas donde se escribe de derecha a izquierda (como el hebreo o el árabe) lo hacen de la manera contraria: el pasado a la derecha y el futuro a la izquierda. Los hablantes de chino mandarín, que se puede escribir de manera vertical (de arriba a abajo) sitúan el pasado arriba y el futuro abajo. Existen por lo tanto multitud de ejemplos de cómo la cultura y la lengua interactúan con la cognición para establecer una estructuración dada. 


\section{L.H.V.: La idea de corporeización e integración de nuestros conceptos,} ¿Ha aclarado varias hipótesis sobre nuestra manera de pensar y elaborar el lenguaje o, después de todo, abre desafíos aún más intrincados para comprender estos procesos?

J.V.M.: Como suele suceder con muchas teorías científicas, ambas cosas son ciertas. Por un lado, la idea de corporeización ha permitido aclarar conceptos hasta ahora muy huidizos: el concepto de "significado" era algo muy etéreo y vago, que súbitamente ha podido ser "capturado", al menos parcialmente. Tenemos ahora una idea muchísimo más concreta de cómo funciona el significado. La teoría de la corporeización y de simulación proporciona una manera muy definida, medible y entendible de acercarse a la semántica. De esta manera, un hablante entiende el significado de una expresión lingüística cuando se produce una reactivación cerebral selectiva de las distintas áreas modales del cerebro que se activaron en la interacción con los referentes de la expresión lingüística. Autores como Glenberg han mostrado de manera innegable que entender una frase como "abre el cajón" consiste en reactivar los circuitos motores implicados en la acción de abrir un cajón, así como el resto de información sensotomora (aspectos visuales, como la forma de un cajón, táctiles, etc.) Existen multitud de experimentos que han mostrado estos procesos de simulación: entender la palabra "limón" implica la reactivación parcial de distintas áreas cerebrales, de manera que se activa una simulación parcial con el color amarillo del limón, su sabor ácido, su forma y tamaño, etc. La evidencia empírica apunta de manera abrumadora a este tipo de procesos de simulación en la cognición: ya se ha demostrado la presencia de muchos aspectos específicos sensomotores, como la forma de los objetos, su movimiento, su color, olor, sabor, el sonido que producen, aspectos propioceptivos, de perspectiva espacial... estas simulaciones incluyen no sólo aspectos sensomotores sino también introspectivos: parte del significado de "serpiente" tiene que ver con la típica sensación de temor o rechazo al interactuar con este animal. Esto hace que aspectos como la connotación o los aspectos emocionales del lenguaje puedan ser incluidos en este acercamiento y se vean igualmente "concretizados", como decíamos antes.

L.H.V.: ¿Y en cuanto a los desafíos? Pareciera ser que a mayor avance, mayor el campo desconocido que se abre al investigador.

J.V.M.: Este acercamiento abre una gran serie de interrogantes que hay que ir contestando poco a poco. Por ceñirnos al campo de la lingüística, 
es especialmente interesante saber cómo se relacionan los distintos aspectos gramaticales con los elementos presentes en una simulación mental. Las simulaciones evocadas por las expresiones lingüísticas son muy sensibles al contexto; cada vez que escuchamos una determinada palabra, se activará un parte distinta de su significado. Predecir qué parte es muy complicado. También es complicado saber cómo la presencia de varias palabras juntas modula el significado (es decir, la simulación) de ambas. Un ejemplo sencillo: la palabra "piano" evoca un determinado sonido en una oración como "desde mi ventana, se escucha el piano del vecino"; en cambio, evoca un objeto físico en una oración como "este piano lo tuvieron que subir cuatro hombres por las escaleras". Otro ejemplo: si alguien tiene un "coche rojo", pensamos en un color distinto del rojo que se activa al decir que alguien tiene "el pelo rojo". Se sabe que el aspecto gramatical del verbo influye en cómo se simula la acción descrita. Cuestiones más sutiles, como la influencia en el distinto orden de los constituyentes, por poner un ejemplo, están todavía por explorar.

En otro orden más general de cosas, también hay que saber el alcance de los procesos de simulación. Parece haber un consenso en autores de distintas persuasiones en que no siempre realizamos simulaciones corporeizadas. En determinadas tareas puede haber otro tipo de mecanismos, de corte más estadístico, simbólico o amodal. Establecer cuándo se utiliza un tipo de mecanismo u otro es otra de las metas más acuciantes del momento.

L.H.V.: ¿Qué se ha descubierto hasta el momento sobre la compleja corporeización de conceptos abstractos? ¿La comporeización indirecta es concluyente como explicación?

J.V.M.: La corporeización de los conceptos abstractos es verdaderamente el caballo de batalla de los estudios de corporeización. Por un lado, es cierto que se han descubierto casos de corporeización indirecta en dominios abstractos. Un caso paradigmático es el tiempo: realmente para procesar expresiones temporales invocamos la ayuda de representaciones espaciales, tal y como se muestran en las expresiones lingüísticas (e.g., "tenemos un gran futuro por delante"; o "dejamos atrás malos momentos"). En distintos experimentos se ha demostrado que el dominio de la moralidad se conceptualiza con base al concepto físico de limpieza, la importancia por medio del peso o el tamaño, las relaciones de control por medio de la verticalidad, la felicidad y la tristeza también 
se organizan verticalmente (felicidad-arriba, tristeza-abajo), la similitud como cercanía física, lo bueno y malo se organizan como arriba-abajo, cerca-lejos o blanco-negro, según los casos y un largo etcétera. Sin embargo, todas estas proyecciones corresponden a lo que se conoce en la lingüística cognitiva como "metáforas primarias", de tipo correlacional. Existe mucha menos evidencia para otro tipo de metáforas más complejas, no correlacionales, como "el amor es un viaje" o "las ideas son comida". También existen dudas de en qué casos se realizan estas conexiones entre dominios (es decir, en qué tipo de tareas). Últimamente, los acercamientos de corte estadístico, como los estudios de Análisis de Semántica Latente (o en inglés, Latent Semantic Analysis, LSA), han mostrado el gran poder de codificación de los patrones combinatorios, estadísticos, en el lenguaje. Como decía anteriormente, hay tareas en las que no hace falta realizar ninguna simulación corporeizada. En general, la influencia del lenguaje sobre la conceptualización de los dominios abstractos está siendo explorada. Existen además más casos documentados en los que no han aparecido las conexiones entre dominios que según la teoría deberían aparecer, lo que evidencia la necesidad de alcanzar modelos más refinados.

\section{4. Últimas investigaciones y Laboratorio Red Hen Lab}

L.H.V.: ¿Cuáles han sido las últimas áreas de investigación que ha desarrollado? ¿Qué evidencias significativas han surgido?

J.V.M.: Sigo intentando avanzar en la construcción de un modelo algo más preciso de las proyecciones conceptuales entre dominios. Para ello, estoy examinando dos dominios abstractos más o menos clásicos: el del tiempo y el de las emociones. Sigo utilizando una mezcla de metodologías, tanto de corpus como experimentación psicolingüística. El modelo lakoffiano más clásico afirma, más o menos, que existen dominios meta que se estructuran por medio de proyecciones de información de un dominio fuente, y estas proyecciones se almacenan en nuestra memoria a largo plazo como parte de nuestro conocimiento del dominio meta. Sin embargo, este modelo tiene ciertos problemas para explicar determinados comportamientos. La evidencia psicolingüística muestra que, cuando se producen (puesto que no siempre aparecen esas proyecciones de manera automática: dependen en gran medida de la tarea que estemos realizando) existe una enorme flexibilidad en esas proyecciones. 


\section{L.H.V.: ¿Qué explicaría, entonces, esa flexibilidad?}

J.V.M.: En el artículo que publiqué en la revista Cognitive Science con el grupo de psicología experimental de Julio Santiago de la Universidad de Granada, se mostraba que las proyecciones dependían en gran medida de la activación de distintos elementos mediante la fijación de la atención: dependiendo de qué esté activado atencionalmente va a aparecer un tipo de proyecciones u otras. En ese artículo mostrábamos cómo un experimento clásico, en el que se mostraba la activación automática del espacio (la verticalidad) en el proceso de términos con una valencia (mediante la cual la palabra "bueno" se lee más rápido en la parte superior de la pantalla, y la palabra "malo" en la parte inferior), depende de si nuestra atención está dirigida a esa dimensión vertical. También demostramos cómo manipulando la atención de los sujetos de la manera adecuada se pueden lograr proyecciones que vayan en el sentido "inverso": en vez de influir la verticalidad en la valencia, logramos que la valencia influyera en nuestros juicios de verticalidad. Estos resultados, junto con muchos otros, son difíciles de explicar desde una concepción rígida de la metáfora en la que existen unas proyecciones "fijas" que se activan de manera automática al procesar la información del dominio meta. Más bien, apuntan en la dirección de un mayor grado de dinamismo y flexibilidad.

\section{L.H.V.: ¿Cómo afectan estos resultados a la teoría de los dominios?} ¿Estaría acaso reconfigurando su conceptualización?

J.V.M.: Si esto es cierto, tendríamos que cambiar nuestra concepción de cómo se estructuran los dominios conceptuales y también de qué es una metáfora, que pasaría a ser un proceso dinámico y emergente más que una conexión mental fija (Lakoff en su teoría de NTL apunta incluso a conexiones físicas, a nivel neuronal). En otro experimento que saldrá publicado en este año 2015, hemos mostrado cómo las conexiones entre dominios pueden ser multidireccionales. En el experimento realizado, pedimos a los sujetos que leyeran un poema de Octavio Paz en el que se utiliza una imagen compleja y altamente poética donde aparece una serpiente como metáfora del paso del tiempo. Normalmente nos imaginamos las serpientes de manera curvada, mientras que el discurrir del tiempo es una línea recta (la línea mental temporal o timeline). Pues bien, aquellos sujetos que comprendían la metáfora temporal tendían a imaginarse la serpiente en una posición más recta, mientras que los que no captaban la metáfora, la imaginaban más curvada. Esto es 
muy sorprendente, porque es un ejemplo de cómo el dominio meta (el tiempo) afecta la representación mental del dominio fuente (la posición espacial de la serpiente). Es decir, el flujo de información va al revés de lo esperado en la teoría clásica. Estos resultados se pueden explicar con otras teorías más flexibles, como la teoría de la integración conceptual de Turner y Fauconnier, pero no con el modelo de Lakoff. En una variante de este experimento pudimos manipular la curvatura con la que los sujetos imaginaban el objeto fuente (la serpiente) introduciendo sesgos atencionales ("facilitación" o "priming") de uno u otro dominio, demostrando de esa manera que el flujo de información en esos casos va desde el tiempo al espacio y no al revés. Alternativamente, estos resultados podrían valer como validación de la existencia de un espacio intermedio, de "amalgama" o "integración conceptual" (blending).

L.H.V.: De acuerdo a su participación en el Red Hen Lab ¿Por dónde han transitado sus estudios en esta colaboración? ¿Qué ventajas significativas implica un proyecto como este para la investigación cognitiva?

J.V.M.: El Red Hen Lab es un laboratorio para la investigación de la comunicación multimodal, integrado por un gran número de investigadores (incluyendo a gente como Mark Turner, el creador junto a Gilles Fauconnier de la teoría del blending, Francis Steen, de la Universidad de los Ángeles o Cristóbal Pagán Cánovas, de la Universidad de Navarra); trabajamos con una enorme base de datos multimodal compuesta de unas 250.000 horas de televisión con subtítulos: el programa anota las marcas temporales de los subtítulos de manera que podemos buscar en el texto compuesto por esos subtítulos como en un corpus textual normal, pero los resultados de la búsqueda nos llevan al archivo multimodal en el que aparece la persona diciendo la expresión lingüística concreta, lo que nos permite estudiar aspectos no sólo textuales (como sería el caso de un corpus normal) sino también multimodales, por ejemplo: la gestualidad, la prosodia, la expresión facial, el contacto visual, etc. Hemos estado utilizando esta poderosa herramienta para ver cómo conceptualiza la gente el dominio del tiempo cuando están hablando de manera espontánea. Para ello, hemos buscado una gran cantidad de expresiones temporales y hemos examinado qué tipo de gesto realiza la gente al decirlos. En el lenguaje (el estudio está realizado en inglés, pero los resultados serían similares para el español, pues se comporta de manera similar) tan sólo existe la posibilidad de organizar el tiempo 
espacialmente utilizando el eje sagital (es decir, de delante a atrás, de manera que el futuro está delante de nosotros y el pasado a nuestra espalda, como en las expresiones "tenemos por delante unos días de mucho trabajo" o "hemos dejado atrás los malos momentos"). No existe, sin embargo, la posibilidad lingüística de organizar el tiempo utilizando el eje lateral (es decir, de izquierda a derecha o al revés, como decir "el mes de la izquierda" para referirnos al mes pasado). Como comentamos anteriormente, estudios psicolingüísticos han probado que la gente sí que utiliza este eje lateral, que se organiza en la dirección de la lecturaescritura. Los hablantes de culturas que escriben de izquierda a derecha (como el inglés o el español) sitúan el pasado a la izquierda y el futuro a la derecha y los de culturas que escriben de derecha a izquierda (como el hebreo o el árabe) al revés: para ellos el pasado está a la derecha y el futuro a la izquierda.

\section{L.H.V.: ¿Estos descubrimientos resultan representativos o} correspondientes con el actuar cotidiano o el "mundo real"?

J.V.M.: Todos estos resultados, muy sólidos, han sido obtenidos en laboratorios, lo que introduce la duda de si está organización es, como dicen los psicólogos un "artefacto de la tarea", es decir, se organiza así en el laboratorio porque la tarea requiere que usemos ese eje lateral, pero no se utiliza de manera espontánea en la vida real. Nuestro estudio demuestra que esta organización espacial es completamente real: tenemos cientos de ejemplos en los que los hablantes gesticulan moviendo sus manos de izquierda a derecha mientras pronuncian expresiones como "de principio a fin" (from start to finish), mostrando que aunque lingüísticamente el eje lateral no se mencione, conceptualmente sí lo están utilizando para la representación del tiempo. Además, nuestros resultados muestran de nuevo un alto grado de flexibilidad: el uso de la línea temporal lateral no es tan fijo como se podría esperar. En muchos casos, los hablantes (de inglés) no utilizan la dirección esperada, sino la contraria y gesticulan indicando un paso del tiempo de derecha a izquierda. Esto ocurre en un porcentaje de casos lo suficientemente numeroso como para ser descartado por irrelevante.

\section{L.H.V.: ¿Y cuáles serían las causas?}

J.V.M.: Factores como si el hablante es diestro o zurdo, la posición relativa de hablante u oyente o incluso la posición de la cámara (recordemos que esto son expresiones utilizadas en programas de televisión), afectan de manera contextual y sensible el tipo de configuración espacial que 
utilizamos. Todo esto apunta, de nuevo, a un modelo mucho más dinámico y flexible que el modelo más clásico utilizado en los estudios de metáfora conceptual. Los planes son extender estos estudios al español, y también averiguar con mayor precisión cuáles son los factores que influyen en que utilicemos una configuración espacial concreta para organizar el tiempo.

L.H.V.: ¿Qué otras ventajas científicas presenta este programa de la UCLA para las ciencias cognitivas? ¿Se ha llegado a otras conclusiones relevantes?

J.V.M.: Como he apuntado anteriormente, el laboratorio Red Hen se dedica a la investigación de la multimodalidad en el lenguaje, que es un aspecto que ha sido muy poco explotado. Yo soy un gran partidario del uso de corpus en los estudios lingüísticos: el corpus es seguramente la herramienta más revolucionaria de los últimos años, y permite sacar a la luz patrones y regularidades hasta ahora no accesibles. Por hacer un símil, el corpus es para los lingüistas como el microscopio para los biólogos: te permite ver cosas que a simple vista básicamente son imposibles de percibir. Sin embargo, la limitación de la mayoría de corpus actuales es su carácter estrictamente textual, puesto que no incluyen datos multimodales. Y este es un aspecto de enorme importancia: los estudios de adquisición de lenguaje, por ejemplo, han mostrado cómo los niños aprenden el lenguaje utilizando estas pistas multimodales. Por ejemplo, los niños son capaces de detectar la dirección de la mirada del interlocutor y la utilizan para realizar sus hipótesis sobre cuál es el referente de una nueva palabra que ellos no conocen aún. En general, gran parte de la comunicación lingüística tiene que ver con procesos atencionales: el lenguaje es una señal para que prestemos atención a algo y para esto, nos debemos de ayudar de muchas pistas multimodales.

L.H.V.: En la rutina diaria es clave la comunicación no verbal, naturalmente.

J.V.M.: El lenguaje real, en su uso cotidiano, es en realidad multimodal y siempre tiene lugar en un contexto dado. Fenómenos como la ironía son difíciles de estudiar de manera únicamente textual, e incluso aspectos tan básicos como el alcance de una negación (saber qué parte de una oración se está negando) en una oración es difícil de establecer por medios únicamente morfosintácticos (para saber qué parte de la oración se está negando en "yo no llamé ayer a tu hermano" hay que recurrir a la entonación o aspectos multimodales). Hasta ahora el estudio de los 
aspectos multimodales tenía una serie de desafíos, empezando por el modo de recoger los datos. Esta parte es la que Red Hen y la base de datos NewsScape nos puede ayudar a mejorar. Pero sigue haciendo falta un modelo integrador de todas estas fuentes de información dispersa. El modelo más prometedor es la gramática de construcciones: hasta ahora, los aspectos formales de la construcción que se han tenido en cuenta han sido los morfosintácticos. Algunos estudios (no muchos, pero sí un número significativo) han incluido información entonativa como parte del polo formal de la construcción. No hay en realidad ningún tipo de incompatibilidad teórica por la cual no podamos ampliar la información asociada a una construcción, incluyendo en ese polo formal información prosódica, gestual, de expresión facial, etc.

\section{L.H.V.: ¿Debemos entonces abogar por modelos de análisis más complejos?}

J.V.M.: Sí, claramente esta información multimodal tiene sus propias peculiaridades: la frase "no lo sé" va frecuentemente asociada con el gesto de encoger los hombros o mover la cabeza lateralmente, pero no siempre. En este sentido, hay que trabajar mucho en un modelo más elaborado que detalle cómo se relaciona esta información multimodal con el contenido textual de la oración, cómo la modula. Esto es claramente un desafío. Los estudios de la metáfora se pueden ver por supuesto también beneficiados por una visión más amplia del fenómeno lingüístico, que vaya más allá de lo puramente textual e incluya el comportamiento real de los participantes en un acto comunicativo y la información proporcionada por estos, por medio de toda clase de pistas. El laboratorio Red Hen está trabajando en conjunción con ingenieros de procesamiento de imagen y de sonido, de manera que podamos identificar de manera automática a determinados hablantes (por ejemplo, identificar a Obama o al Dalai Lama), clasificarlos según sean hombres o mujeres, o etiquetar de manera automática la emoción asociada a una determinada expresión o el tema del que se está hablando. Esto nos permitiría hacer búsquedas del tipo "encuéntrame ejemplos de personas del sexo femenino hablando del remordimiento, que muestren tristeza y hagan gestos laterales con las dos manos", por poner un ejemplo. La idea de Red Hen es anotar la base de datos multimodal con sucesivas "capas" de etiquetas, morfosintácticas, semánticas, prosódicas, gestuales, temáticas, etc., que permitan cruzar datos de distintas fuentes de información. La combinación de esta rica y variada fuente de información con modernas técnicas de corpus puede 
dar resultados que nos van a sorprender. Un corpus es un poco como un niño: nunca sabes muy bien qué se le va a ocurrir decir. Ahora mismo tenemos una cantidad de datos sobre la conceptualización del tiempo que van a hacernos replantearnos las explicaciones que hemos estado utilizando hasta el momento. Y esto no ha hecho más que empezar: realmente, el proyecto está en sus fases más iniciales. Es de esperar que en los años siguientes obtengamos una enorme cantidad de datos que, necesariamente, nos forzarán a refinar nuestros modelos explicativos, algo siempre deseable para cualquier ciencia.

\section{Desafíos futuros}

L.H.V.: Aparte de las concepciones del tiempo y el espacio, ¿Qué otros tópicos debieran abordarse, por lo pronto, en los estudios de la metáfora? J.V.M.: Aunque no creo que sea posible establecer un listado exhaustivo de dominios abstractos, claramente existen una gran cantidad de ellos, la mayoría todavía sin estudiar. Actualmente, se han estudiado con más atención los casos en los que un dominio sensomotor muy básico y concreto se utiliza para estructurar algún otro. El dominio más paradigmático es el del espacio, que se ha estudiado en relación con el tiempo, pero también con otro tipo de dominios, como la similitud, la valencia emocional, las relaciones de poder, las variaciones cuantitativas, etc. También se han estudiado otros dominios fuentes concretos como el peso (para estudiar el dominio de la importancia) o el cromático (como la relación entre blanco-negro y bueno-malo). El modo de proceder de los estudios de metáfora puede ir en ambas direcciones: se pueden tomar dominios fuente para ver su influencia en dominios abstractos o bien centrarse en dominios abstractos y ver qué mecanismos se utilizan para estructurarlos. Por poner un ejemplo muy evidente, creo que el dominio de las emociones es de los más importantes e influyentes: ha habido una revolución en las ciencias cognitivas sobre la gran influencia de los procesos emocionales en la cognición, de manera que ya no se aboga por esa separación clara entre emoción-razón. La conceptualización de nuestras emociones es un tema muy interesante y de gran importancia en el que, de nuevo, se encuentran factores más universales de tipo biológico junto con factores de tipo más cultural, entre los que se cuenta el lenguaje, por supuesto. En este sentido, es cierto que hay mucho trabajo hecho, pero todavía queda mucho por hacer. 
En mi opinión, una de las metas más acuciantes de los estudios de metáfora, incluso más aún que la búsqueda de nuevos aspectos a estudiar, es su evolución hacia una mayor solidez como disciplina. Para esto, es básico avanzar en cuestiones metodológicas. El objetivo debería ser la integración de estudios de corte más observacional o intuitivo con la gran panoplia de métodos de la ciencia cognitiva. Por ejemplo, se deberían establecer técnicas y procedimientos generalizados que sirvieran como estándares en los estudios de corpus, especialmente aquellos que van más allá del acercamiento inicial de "corpus como búsqueda de ejemplos" para intentar acercarse a un mayor nivel de sofisticación que permita la validación estadísticas de hipótesis concretas. En general, habría que tomarse en serio el camino de la "evidencia convergente", aunando estos estudios con otros como los estudios psicolingüísticos de tiempo de reacción, los estudios de movimientos oculares, estudios procedentes de la neurociencia, etc. La idea es poder tener, como ya ocurre en otras disciplinas científicas, un listado de herramientas a nuestra disposición que permitan extraer información, validar nuestras hipótesis y comparar nuestros resultados. Es decir, incorporar los estudios de metáfora a otras disciplinas "acumulativas", en las cuales los resultados previos y la discusión entre investigadores permiten avanzar en una dirección, mejorar y refinar los modelos explicativos.

\section{L.H.V.: Es decir, de nuevo deberíamos abordar las teorías de la metáfora de manera más abierta y flexible.}

J.V.M.: Es nuestro deber como investigadores mantener una mente abierta para refinar nuestros modelos, considerando nuevas posibilidades de manera más aséptica y neutra, intentando no dejarse arrastrar por seguidismos de un acercamiento teórico concreto. El objetivo no debería ser intentar encontrar evidencia de que la teoría $\mathrm{X}$ o la teoría $\mathrm{Y}$ son correctas, sino averiguar cómo funcionan las cosas. Personalmente, me gustaría ver una mayor relación entre las explicaciones corporeizadas y los ya mencionados procedimientos estadísticos combinatorios, en el que los patrones de co-ocurrencia entre palabras permiten la inducción de una determinada conceptualización. Creo que el papel del lenguaje ha sido subestimado en la creación de patrones de conceptualización metafórica y estos acercamientos pueden ser una manera de cerrar esta separación. 


\section{L.H.V.: Desde las neurociencias se ha pretendido alcanzar avances} que puedan descubrir la correspondencia específica entre unidades discursivas, como las metáforas, con unidades neuronales en el cerebro, ¿Llegaremos a ese nivel de exactitud?

J.V.M.: La verdad es que no creo que ese nivel de exactitud sea posible, en primer lugar porque el funcionamiento de estos asuntos no es tan sencillo. Más bien al contrario, debe ser extremadamente complejo, con una gran cantidad de elementos involucrados, distribuidos por distintas zonas cerebrales. En este sentido, tengo que expresar mi escepticismo frente a propuestas como la de la Neural Theory of Language que describen las metáforas como conexiones neuronales entre distintas áreas cerebrales. Hay muchas razones para pensar que esto es una simplificación excesiva. Para empezar, una nota extremadamente escéptica: aunque sabemos cómo funciona una neurona aislada, qué es lo que pasa cuando funcionan unos pocos miles de neuronas juntas es mucho más misterioso. Pero incluso así, existen muchas razones para pensar que estamos aún lejos de un modelo neuronal del funcionamiento metafórico, desde la gran plasticidad del cerebro, que permite una gran variabilidad inter-sujeto (e incluyo intrasujeto), y la complejidad del comportamiento a modelar, que incluye relaciones con muchos sistemas interconectados: el lingüístico, los sistemas atencionales, los emocionales, la base corporeizada, el conocimiento cultural. Todavía nos falta tener una idea más clara de cómo interactúan todas estas piezas: el tener un modelo más claro, preciso y elaborado es más urgente que saber hasta el último detalle de su implementación física. Lo que no quiere decir que no se pueda ir avanzando en el camino. Posiblemente, algunas de las piezas de este rompecabezas se puedan ir relacionando con aspectos neuronales concretos, en el espíritu de evidencia convergente que mencionábamos anteriormente. En resumen, que no creo que sea preciso llegar hasta la última neurona para poder avanzar en nuestro conocimiento de cómo funcionan las cosas.

\section{Conclusión}

El Dr. Javier Valenzuela da cuenta de la indiscutible solidez de la lingüística cognitiva en los estudios del lenguaje y su irreprochable aporte para las ciencias cognitivas en general, involucrando investigadores más allá del área verbal. Además, son múltiples las asociaciones, revistas, 
publicaciones, textos e investigaciones que en décadas la han constituido en un paradigma más que válido, que supera los cuestionamientos, hoy infundados, sobre una trayectoria que se manifiesta bastante consolidada.

Para el entrevistado, la interacción entre mente, cuerpo y ambiente es clave. En ese sentido, refiere que de alguna manera se deben integrar la dicotomía entre innatismo y factores externos (transformada en un clásico gracias a Chomsky y Foucault), considerando una mirada del tipo "el cerebro propone y la lengua/cultura escoge", lo cual explicaría de mejor manera el fenómeno de la construcción de significados. No se puede obtener una concepción del fenómeno lingüístico complejo sin realizar aproximaciones que sean medianamente multimodales: implicando lo no verbal, la experiencia, el cuerpo, la emoción, el sonido, etc.

Actualmente, se han detectado importantes avances en la teoría de dominios, mostrándose más flexible en su conformación de lo que anteriormente se conocía, siendo un campo que requiere suma labor para afinar los modelos de estudio que pretenden detectar qué sucede específicamente en las actividades cerebrales frente a las expresiones lingüísticas. En esa línea, analizando otras propuestas investigativas, el autor indica que estamos muy lejos de poder configurar un modelo neuronal de, por ejemplo, el funcionamiento metafórico, que es uno de los desafíos trascendentales que han planteado neurolingüistas de la talla de David Poeppel.

Sin duda, la propuesta de Red Hen Lab ha permitido acceder a una base de datos privilegiada para realizar no sólo estudios verbales, sino también no verbales, y cómo se integran en una compleja red multimodal de uso real, salvando el riesgo de generar investigaciones a partir de "artefactos de la tarea" que distorsionan el fenómeno que se pretende estudiar.

Finalmente, para el profesor Valenzuela el poder afinar el estudio de la metáfora como una disciplina en sí misma, perfeccionando las metodologías de investigación, es uno de los desafíos más importantes en la lingüística cognitiva en general y en su particular y próximo horizonte científico. 


\section{Bibliografía}

Dolscheid, S., Shayan, S., Majid, A., \& Casasanto, D. (2013). The thickness of musical pitch: Psychophysical evidence for linguistic relativity. Psychological Science, 24(5), 613621 